\title{
Traduire
}

Une autre perspective sur r tr traduction

Revue française de la traduction

$235 \mid 2016$

Luxe, mode... et traduction !

\section{Chat alors!}

Lakshmi Ramakrishnan lyer

\section{(2) OpenEdition}

Journals

Édition électronique

URL : https://journals.openedition.org/traduire/860

DOI : $10.4000 /$ traduire.860

ISSN : 2272-9992

Éditeur

Société française des traducteurs

Édition imprimée

Date de publication : 1 décembre 2016

Pagination : 91-93

ISSN : 0395-773X

\section{Référence électronique}

Lakshmi Ramakrishnan lyer, « Chat alors! », Traduire [En ligne], 235 | 2016, mis en ligne le 01

décembre 2018, consulté le 06 juillet 2021. URL : http://journals.openedition.org/traduire/860 ; DOI :

https://doi.org/10.4000/traduire.860 


\section{Chat alors !}

\section{Lakshmi Ramakrishnan lyer}

Une vérité universellement reconnue est que la traductrice pourvue d'informations hétéroclites glanées au gré des textes qu'elle traite doit être une redoutable joueuse de Trivial Pursuit. Quant à celle qui baigne dans le Luxe et ses affluents (la Fine Gastronomie et la Haute Couture, pour n'en citer que deux), elle brillera également dans les dîners en ville. Quelle assemblée de catophiles chics ne serait pas ravie d'apprendre, par exemple, cette information capitale tirée d'un reportage sur un palace parisien ? II se trouve que deux sacrés de Birmanie séjournent au Bristol à l'année. Ces deux Aristochats se prénomment Fa-Raon et Kléopatre ; ils arborent chacun un collier créé par la Maison Goyard, assorti d'un médaillon signé Christofle.

"Mais c'est indécent ", protestera une convive à l'âme sensible et à la consommation strictement éthique, "quand il y a tant d'êtres humains qui souffrent ". Et voilà qu'un débat animé se lance, pour le plus grand plaisir de Madame la maîtresse de maison, qui se délecte d'avance des commentaires élogieux sur sa page Facebook au lendemain de sa soirée réussie. Décidément, il faudra réinviter cette traductrice !

Je ne suis pas immergée dans l'univers du luxe au jour le jour, mais il m'arrive d'y faire trempette, à travers des textes pour des revues lifestyle (anciennement connu sous l'appellation " art de vivre ") visant un lectorat haut de gamme. Faute de mode d'emploi véritable, je vous livre ici quelques réflexions - en vrac, purement personnelles, et plus ou moins pertinentes.

II peut y avoir une certaine dichotomie entre l'opulence de ladite revue lifestyle et la pitance proposée à celui ou celle qui la traduira. II vous arrivera plus d'une fois d'entendre l'affirmation suivante : "Le tarif, dans la presse, c'est 8 centimes le mot ". Tenez bon. Imposez vos prétentions. Ce tarif-là ne pourra en aucun cas subventionner votre lifestyle à vous, qui vivez d'amour et de champagne (très) frais.

Comme si souvent dans notre beau métier, vous ne disposerez pas, aux yeux du donneur d'ouvrage, d'un nom et d'un prénom. Vous serez la "traductrice ". En copie des échanges de 
courriels entre client, contributeurs, maquettiste et autres, vous vous verrez nommée ainsi à plusieurs reprises : la " traductrice ". Ce n'est pas la peine de vous offusquer. Saisissez plutôt cette occasion pour sortir de l'anonymat. Pointez-vous dans le bureau du client, armée de votre plus beau sourire, lors de votre prochain passage dans ses beaux quartiers, et faites sa connaissance de visu. Le must en termes d'accessoire pour cette visite de courtoisie ? Un chien, labrador(able) de préférence. Même si votre client peinera toujours à se souvenir de votre patronyme, au moins serez-vous devenue, dans son esprit, la " traductrice au toutou ", ce qui marquera votre singularité et vous distinguera du confrère ou de la consœur lambda.

Côté contenu, la revue lifestyle a quelques particularités. Le texte n'est que prétexte (le véritable intérêt de la publication étant de nature iconographique) mais il doit " faire joli ", tout autant que l'image. De ce fait, il sera souvent parsemé de citations d'œuvres jamais traduites, de jeux de mots sans équivalent, de références trop franco-françaises pour un lectorat que l'on veut (mais l'est-il vraiment?) international, et de descriptions endimanchées.

Ainsi, jaillissant de la souris d'un habitué des établissements Relais \& Châteaux : " On m'avait annoncé des aubes somptueuses. Du coup, pendant toute la nuit, j'ai guetté le moucheté de la pénombre. L'aurore approcha, déposa ses à-plats, aubergines, ses hémorragies de gris étain, de violets inédits, d'orangés Hermès "(1). [l'd been told of sumptuous dawns. And so I spent all night peering into the mottled darkness. Then sunrise crept up, filling the sky with swatches of aubergine, pewter gray, unexpected lilac and Hermès orange].

La rencontre avec le client servira, entre autres, à expliquer que vous allez traduire " librement ", adapter son contenu, ajouter du contexte. Le petit panégyrique sur La Colombe d'Or, auberge de luxe provençal ("L'ami des artistes où les nuits s'échangent contre des toiles. Simone et Yves. ") n'aurait hélas pas de sens immédiat pour le lecteur étranger. Vous serez obligée de sacrifier poésie et concision, d'opter pour le terre-à-terre [The artist-friendly auberge where room and board could once be had in exchange for a canvas. Simone (Signoret) and Yves (Montand) met here].

Côté matériel de référence, le dictionnaire synonymique sera un précieux allié. Mention spéciale au Dictionnaire des synonymes, mots de sens voisin et contraires pour ceux qui travaillent, non seulement vers, mais aussi depuis le français : les nuances d'un mot dans la langue source peuvent aider à trouver une nuance correspondante dans la langue d'arrivée. C'est dans Gabay's Copywriters Compendium que j'ai trouvé un équivalent parfait de l'expression « aimable

(1) SIMON François. 
comme une porte de prison " [about as friendly as a shark with toothache]. Pour les citations, la recherche dans Google Books peut sauver la situation s'il s'agit de l'œuvre d'une Colette, plus communément (quoique pas entièrement) traduite qu'une Louise (de Vilmorin). Ah, les sueurs froides qu'elle m'a occasionnées, cette chère Louise ! Vous vous en doutez, je me garderais bien de vous livrer mes tentatives de traduction. En revanche... vous prendrez bien un peu de bibliographie?

lakshmi.iyer@mac.com

Traductrice libérale et d'édition du français et de l'italien vers l'anglais depuis 2004, Lakshmi Ramakrishnan lyer travaille principalement dans les domaines des arts et de la culture.

\section{Bibliographie}

BERTAUD DU CHAZAUD Henri, 2007, Dictionnaire de synonymes, mots de sens voisin et contraires, Paris, Gallimard.

GABAY Jonathan J., 2006, Gabay's Copywriters' Compendium, Oxford (Grande-Bretagne), Elsevier Butterworth-Heinemann.

DE BLAYE Diane et EFRATAS Pierre, 1997, Passez-moi l'expression en anglais, Paris, Belin. HAMILTON Grant et LAVALLÉE François, 2012, Tweets et gazouillis pour les traductions qui chantent, Montréal (Canada), Linguatech.

LES GROSSES VOITURES DE SPORT ATTIRENT LES FEMMES

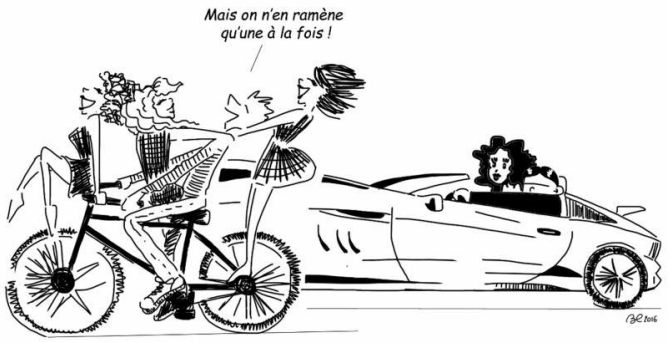

